\title{
Cianogênese em esporófitos de pteridófitas avaliada pelo teste do ácido pícrico ${ }^{1}$
}

\author{
Marcelo Guerra Santos ${ }^{2,7}$, Carlos Eduardo Martins Carvalho ${ }^{3}$, Alphonse Kelecom ${ }^{4}$, \\ Maria Luiza Ribeiro da Costa Ribeiro ${ }^{5}$, Camila Vaz Chagas de Freitas ${ }^{5}$, Leonardo Moreira da Costa e $^{6}$ \\ Livia Viana de Godoy Fernandes ${ }^{6}$
}

Recebido em 07/01/2004. Aceito em 12/04/2005

RESUMO - (Cianogênese em esporófitos de pteridófitas avaliada pelo teste do ácido pícrico). Os glicosídeos cianogênicos são substâncias de defesa encontradas em alguns vegetais, capazes de liberar ácido cianídrico por meio de reações de hidrólise. O presente estudo teve como objetivo avaliar a ocorrência da cianogênese em esporófitos de pteridófitas num afloramento rochoso localizado no Parque Estadual da Serra da Tiririca, Rio de Janeiro, Brasil. Foram analisadas mensalmente durante um ano, folhas férteis, estéreis, jovens (báculos) e caules de 19 espécies de pteridófitas, distribuídas em 13 gêneros e nove famílias. A cianogênese foi caracterizada pelo teste do papel de filtro gotejado com ácido pícrico. Das pteridófitas estudadas, 14 foram pela primeira vez analisadas e 14 espécies apresentaram resultado positivo para a cianogênese. Os resultados encontrados demonstraram haver variação na liberação do ácido cianídrico nas espécies em diferentes partes do vegetal ao longo do período analisado. Microgramma vacciniifolia (Langsd. \& Fisch.) Copel. (Polypodiaceae) e Pteridium aquilinum (L.) Kuhn var. arachnoideum (Kaulf.) Brade (Dennstaedtiaceae) mostraram-se cianogênicas durante todo o período analisado.

Palavras-chave: metabólitos secundários, plantas tóxicas, ecologia química, monilophyta, licophyta

\begin{abstract}
Cyanogenesis in sporophytes of pteridophytes evidenced by the picric acid test). Cyanogenic glycosides are defense substances found in some plants that are able to produce cyanhydric acid on hydrolysis. The present work aimed to study the occurrence of cyanogenesis in pteridophytes of rocky areas of Pedra de Itacoatiara, State Park of Serra da Tiririca, Rio de Janeiro State. Nineteen species, distributed in 13 genera and nine families have been analyzed monthly, during one year. In each species, the analysis was carried out on fertile leaves, sterile ones, young leaves (croziers) and stems. Cyanogenesis was evidenced using filter paper embedded with picric acid. Among studied species, 14 were analysed here for the first time, and 14 gave a positive picric test. Our results showed variation along time in the production of cyanhydric acid in the vegetal species and parts. The species, Microgramma vacciniifolia (Langsd. \& Fisch.) Copel. (Polypodiaceae) and Pteridium aquilinum (L.) Kuhn var. arachnoideum (Kaulf.) Brade (Dennstaedtiaceae) were cyanogenic all along the studied period.
\end{abstract}

Key words: secondary metabolites, toxic plants, chemical ecology, monilophyte, licophyte

\section{Introdução}

As pteridófitas, segundo Tryon \& Tryon (1982), possuem cerca de 9.000 espécies, das quais cerca de 2.250 ocorrem nas Américas. Windisch (1996) estima que 1.150 espécies de pteridófitas ocorram no Brasil. A região sudeste-sul do Brasil possui cerca de 600 espécies e abriga um dos centros de endemismo e especiação de pteridófitas no Continente Sul-Americano (Tryon 1986).

Por meio de muitas formas, as plantas protegem-se contra a herbivoria; apresentando espinhos, acúleos, paredes celulares lignificadas ou silicosas e substâncias químicas de proteção, que podem ser repelentes, impalatáveis, pinicantes ou tóxicas. A seleção e coevolução, em resposta à pressão de predação,

1 Trabalho financiado pela FAPERJ, com o auxílio à infraestrutura (PAI, n. E-26/170.694/2001).

2 Universidade do Estado do Rio de Janeiro, Departamento de Ciências, Faculdade de Formação de Professores, Rua Dr. Francisco Portela 794, CEP 24435-000, São Gonçalo, RJ, Brasil

3 Universidade Federal Fluminense, Instituto de Química, Campus do Valonguinho s/n, CEP 24020-150, Niterói, RJ, Brasil

4 Departamento de Biologia Geral, Laboratório de Química Bio-orgânica, Universidade Federal Fluminense, C. Postal 100.436, CEP 24001-970, Niterói, RJ, Brasil

5 Universidade Federal Fluminense, Instituto de Biologia, Campus do Valonguinho s/n, CEP 24020-150, Niterói, RJ, Brasil

6 Universidade Federal Fluminense, Faculdade de Farmácia, Rua Mário Viana 523, CEP 24241-002, Niterói, RJ, Brasil

7 Autor para correspondência: mguerras@bol.com.br 
resultaram em grande variedade de substâncias de defesa nas plantas, dentre elas, os glicosídeos cianogênicos (Larcher 2000).

Muitas plantas são capazes de sintetizar compostos que liberam ácido cianídrico ( $\mathrm{HCN}$ ) quando o tecido vegetal é injuriado. Estas substâncias podem ser cianoglicosídeos ou cianolipídios que, quando hidrolisados por enzimas, formam açúcares, ácidos graxos, aldeído ou cetona e ácido cianídrico (Buhrmester et al. 2000). A liberação do ácido cianídrico é considerada mecanismo de defesa vegetal (Harborne 1984; Ricklefs 1996) e o seu efeito é a inibição da cadeia respiratória, resultando na morte do herbívoro (Schvartsman 1992). Os glicosídeos cianogênicos são considerados um protótipo químico de substâncias defensivas mais elaboradas, como os alcalóides e, dentre os mais freqüientemente encontrados nas pteridófitas, estão a prunassina e a vicianina (Swain \& Cooper-Driver 1973). Jones (1988) comentou que a cianogênese não é um método efetivo de defesa, e sim um sistema conservativo, visto que os compostos envolvidos são reciclados e a planta precisa ser fisicamente injuriada para a cianogênese ocorrer.

A cianogênese tem sido detectada em pelo menos 2.650 espécies de plantas pertencentes a mais de 130 famílias e tem sido registrada em bactérias, fungos, líquens, pteridófitas, gimnospermas e angiospermas (Buhrmester et al. 2000).

No Brasil, os estudos enfocando a cianogênese são raros, merecendo destaque o trabalho de Kaplan et al. (1983) que realizaram análises em três espécies de pteridófitas (Blechnum serrulatum Rich., Polypodium brasiliensis (= Polypodium triseriale Sw.) e Polypodium sp.). Trabalhos realizados fora do Brasil e que destacaram as pteridófitas foram os de Cooper-Driver \& Swain (1976), Haper et al. (1976), Cooper-Driver et al. (1977) e Hadfield \& Dyer (1988).

O presente estudo teve como objetivo avaliar a ocorrência da cianogênese em pteridófitas de afloramentos rochosos e contribuir para o conhecimento químico do grupo, subsidiando futuros estudos ecológicos.

\section{Material e métodos}

Dezenove espécies de pteridófitas foram coletadas na Pedra de Itacoatiara, situada no município de Niterói entre as coordenadas geográficas de

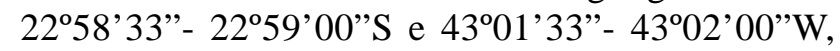
localizada no Parque Estadual da Serra da Tiririca,
Estado do Rio de Janeiro, Brasil. Trata-se de um afloramento rochoso de granito-gnaisse, com cerca de $200 \mathrm{~m}$ de altitude e limitado, de um lado, pela floresta ombrófila densa de encosta e de outro, pelo mar. Essas formações são denominadas "inselbergs" (Porembski 2002). As maiores limitações à instalação da vegetação são o déficit hídrico ao qual é submetida, as altas temperaturas que podem chegar à $50{ }^{\circ} \mathrm{C}$ na superfície da rocha, a reduzida disponibilidade de substratos para as espécies terrestres e a influência da salinidade (Pontes 1987; Meirelles et al. 1999; Porembski \& Barthlott 2000).

As amostras vegetais do caule, folhas jovens (báculo), folhas férteis e estéreis foram coletadas no campo e acondicionadas em sacos de plástico que foram vedados e levados ao laboratório. A análise química foi realizada no mesmo dia ou no dia seguinte, sendo então as amostras guardadas intactas na geladeira. Nas espécies com rizoma, apenas parte deste era retirada (mínimo para análise), sendo a planta preservada, para evitar impacto na flora pteridofítica da área estudada. Foram realizadas coletas mensais no período de agosto/2001 a julho/2002. Por razões técnicas, não houve coleta de material botânico no mês de março/2002.

Cada espécime foi herborizado seguindo as técnicas de Silva (1984) e Windisch (1992), identificado com bibliografia específica e depositado nos Herbários do Jardim Botânico do Rio de Janeiro (RB) e da Faculdade de Formação de Professores da Universidade do Estado do Rio de Janeiro (RFFP, não indexado). O sistema de classificação adotado foi o de Kramer \& Green (1990), com modificações para o tratamento da família Polypodiaceae, reconhecendo-se o gênero Microgramma sensu Tryon \& Tryon (1982).

A detecção da cianogênese foi verificada pela técnica da liberação de ácido cianídrico decorrente da hidrólise enzimática de glicosídeos cianogênicos, utilizando-se uma fita umedecida com solução saturada de ácido pícrico. Primeiramente cada amostra coletada foi colocada dentro de tubo de ensaio e macerada com ajuda de um bastão de vidro, adicionando-se quatro gotas de clorofórmio $(0,3 \mathrm{ml})$. Em seguida, uma fita de papel de filtro gotejada com solução aquosa saturada de ácido pícrico (neutralizada com $\mathrm{NaHCO}_{3}$ e filtrada) foi colocada no interior de cada tubo de ensaio, de modo que não tocasse no material macerado, sendo então cada tubo fechado com uma rolha. A solução de ácido pícrico confere à fita de papel cor amarela. $\mathrm{O}$ resultado é positivo quando a fita adquire coloração laranja-avermelhada. 


\section{Resultados e discussão}

Foram analisadas 2.338 amostras vegetais de 19 espécies de pteridófitas, sendo 421 de caule, 418 de báculos, 860 de folhas estéreis e 639 de folhas férteis. Destas, 232 amostras $(9,9 \%)$ apresentaram resultado positivo para a cianogênese. Das 19 espécies analisadas, 14 foram cianogênicas (Tab. 1), representando $73,7 \%$ das espécies analisadas. Apesar de Harborne (1984) recomendar o método do ácido pícrico em papel de filtro, ele alertou que este teste não é inteiramente específico para a cianogênese, especialmente em espécies nativas de Brassica (Brassicaceae), onde a liberação de isotiocianatos voláteis pode revelar um falso positivo. Este autor também comentou sobre a deficiente sensibilidade do teste e sugeriu a associação deste com o método de Feigl-Anger. Kaplan et al. (1983) justificaram a utilização do teste de ácido pícrico que, segundo eles, revela tendência à cianogênese. Harper et al. (1976) realizaram testes para a cianogênese em 298 pteridófitas cultivadas nas estufas do Royal Botanic Gardens, em Kew e apenas 16 deram resultado positivo $(5,4 \%)$. Estes autores afirmam que a cianogênese não é fenômeno comum entre as pteridófitas. Entretanto, pode haver variação da cianogênese nos indivíduos. No presente estudo observou-se a cianogênese em algumas espécies ainda não estudadas para esta característica (Tab. 1).

As famílias com o maior número de espécies coletadas foram Pteridaceae (cinco espécies), Polypodiaceae, Schizaeaceae e Selaginellaceae (com três espécies cada). Das três espécies de Polypodiaceae coletadas, todas apresentaram resultado positivo para a cianogênese (Tab. 1). Microgramma vacciniifolia e Pteridium aquilinum var. arachnoideum mostraram-se cianogênicas em todo o período analisado, enquanto outras espécies apresentaram-se cianogênicas em apenas um ou dois dos meses do período analisado (Tab. 2). Nessas espécies, a cianogênese pode ser realmente pouco freqüente, ou o resultado positivo pode ser um artefato

Tabela 1. Percentual de meses em que ocorreu cianogênese em esporófitos de pteridófitas coletados na Pedra de Itacoatiara, Niterói, RJ, Brasil, no período de agosto/2001 a julho/2002 (“_” não analisado).

\begin{tabular}{|c|c|c|c|c|}
\hline Família/Espécies & Caule & Báculo & Folha fértil & Folha estéril \\
\hline \multicolumn{5}{|l|}{ ASPLENIACEAE } \\
\hline Antigramma plantaginea (Schrad.) C. Presl* & - & 11,1 & 11,1 & 0 \\
\hline \multicolumn{5}{|l|}{ BLECHNACEAE } \\
\hline Blechnum serrulatum Rich. & 0 & 12,5 & 0 & 0 \\
\hline \multicolumn{5}{|l|}{ DENNSTAEDTIACEAE } \\
\hline Pteridium aquilinum (L.) Kuhn var. arachnoideum (Kaulf.) Brade & 0 & 100,0 & - & 9,1 \\
\hline \multicolumn{5}{|l|}{ LYCOPODIACEAE } \\
\hline Lycopodiella cernua (L.) Pic. Serm.* & 12,5 & - & 12,5 & 11,1 \\
\hline \multicolumn{5}{|l|}{ POLYPODIACEAE } \\
\hline Microgramma vacciniifolia (Langsd. \& Fisch.) Copel. (epífita)* & 27,3 & - & 100 & 100 \\
\hline M. vacciniifolia (Langsd. \& Fisch.) Copel. (rupícola)* & 11,1 & - & 100 & 100 \\
\hline Polypodium catharinae Langsd. \& Fisch.* & 0 & 10 & 0 & 10 \\
\hline P. triseriale $\mathrm{Sw}$. & 0 & 0 & 20 & 9,1 \\
\hline \multicolumn{5}{|l|}{ PTERIDACEAE } \\
\hline Adiantopsis radiata (L.) Fée* & - & 12,5 & 0 & 10 \\
\hline Adiantum raddianum $\mathrm{C}$. Presl & - & 0 & 20 & 0 \\
\hline Doryopteris collina (Raddi) J. Sm. * & - & 0 & 9,1 & 0 \\
\hline D. varians (Raddi) J.Sm. * & - & 0 & 0 & 0 \\
\hline Hemionitis tomentosa (Lam.) Raddi $*$ & - & 0 & 9,1 & 0 \\
\hline \multicolumn{5}{|l|}{ SCHIZAEACEAE } \\
\hline Anemia hirsuta (L.) Sw.* & 0 & 0 & 0 & 0 \\
\hline A. tomentosa (Sav.) Sw. var. anthriscifolia (Schrad.) Mickel * & - & 0 & 0 & 0 \\
\hline A. villosa Humb. \& Bonpl. ex Willd.* & - & 0 & 0 & 9,1 \\
\hline \multicolumn{5}{|l|}{ SELAGINELLACEAE } \\
\hline Selaginella muscosa Spring * & 12,5 & - & 33,3 & 11,1 \\
\hline S. sellowii Hieron. * & 0 & - & - & 0 \\
\hline S. sulcata (Desv. ex Poir.) Spring ex Mart. * & 11,1 & - & 0 & 0 \\
\hline \multicolumn{5}{|l|}{ THELYPTERIDACEAE } \\
\hline Macrothelypteris torresiana (Gaudich.) Ching & - & 0 & 0 & 0 \\
\hline
\end{tabular}

* primeira análise para a presença da cianogênese 
Tabela 2. Percentual mensal de amostras cianogênicas de esporófitos de pteridófitas coletados na Pedra de Itacoatiara, Niterói, RJ, Brasil, no período de agosto/2001 a julho/2002. O mês de março/2002 não foi analisado (“-” não analisado).

\begin{tabular}{|c|c|c|c|c|c|c|c|c|c|c|c|c|}
\hline Espécies & Ago & Set & Out & Nov & Dez & Jan & Fev & Mar & Abr & Mai & Jun & Jul \\
\hline Adiantopsis radiata & 0 & 10 & 0 & 0 & 0 & 0 & 0 & - & 0 & 0 & 20 & 0 \\
\hline Adiantum raddianum & - & 0 & 0 & 20 & - & 0 & - & - & 0 & - & - & 0 \\
\hline Anemia hirsuta & 0 & - & - & - & - & - & 0 & - & 0 & 0 & 0 & 0 \\
\hline Anemia tomentosa var. anthriscifolia & - & 0 & - & - & 0 & 0 & 0 & - & 0 & 0 & 0 & 0 \\
\hline Anemia villosa & 0 & 44.4 & 0 & 0 & 0 & 0 & 0 & - & 0 & 0 & 0 & 0 \\
\hline Antigramma plantaginea & - & 0 & 0 & - & 9,1 & 0 & 0 & - & 0 & 0 & 6,7 & 0 \\
\hline Blechnum serrulatum & 0 & 0 & 0 & 10 & 0 & 0 & 0 & - & 0 & 0 & 0 & 0 \\
\hline Doryopteris collina & 0 & 0 & 0 & 0 & 0 & 0 & 0 & - & 0 & 0 & 7,1 & 0 \\
\hline Doryopteris varians & - & 0 & 0 & 0 & 0 & 0 & 0 & - & 0 & 0 & 0 & 0 \\
\hline Hemionitis tomentosa & 0 & 0 & 0 & 50 & 0 & 0 & 0 & - & 0 & 0 & 0 & 0 \\
\hline Lycopodiella cernua & - & 0 & 0 & - & 0 & 0 & 0 & - & 0 & 0 & 40 & 0 \\
\hline Macrothelypteris torresiana & - & 0 & 0 & 0 & - & 0 & - & - & 0 & - & - & 0 \\
\hline Microgramma vacciniifolia (epífita) & 35,7 & 60 & 53,8 & 64,3 & 26,7 & 53,3 & 60 & - & 20 & 66,7 & 86,7 & 50 \\
\hline Microgramma vacciniifolia (rupícola) & - & 66,7 & 40 & - & 61,5 & 64,3 & 54,5 & - & 38,5 & 41,7 & 91,7 & 58,3 \\
\hline Polypodium catharinae & 0 & 5 & 5 & - & 0 & 0 & 0 & - & 0 & 0 & 0 & 0 \\
\hline Polypodium triseriale & 0 & 0 & 0 & 30 & 12,5 & 0 & 0 & - & 0 & 0 & 0 & 0 \\
\hline Pteridium aquilinum var. arachnoideum & 40 & 33,3 & 13,3 & 80 & 35,7 & 33,3 & 33,3 & - & 28,6 & 26,7 & 33,3 & 33,3 \\
\hline Selaginella muscosa & - & 0 & 0 & - & 20 & 0 & 0 & - & 0 & 0 & 0 & 0 \\
\hline Selagniella sellowii & 0 & 0 & 0 & 0 & 0 & 0 & 0 & - & 0 & 0 & 0 & 0 \\
\hline Selaginella sulcata & 0 & 0 & 0 & 0 & 10 & 0 & 0 & - & 0 & 0 & 0 & 0 \\
\hline
\end{tabular}

do teste do ácido pícrico, sendo necessário estudos complementares. Buhrmester et al. (2000) estudando populações de Sambucus canadensis L. (Caprifoliaceae), registraram variação na produção de compostos cianogênicos, gerando, algumas vezes, dados inconsistentes em determinadas populações. Estes autores especulam que diversos fatores, como hora do dia em que a amostra é coletada, idade das plantas e época do ano em que são coletadas, assim como os efeitos genéticos ou ambientas nas plantas, podem estimular ou não a produção do glicosídeo cianogênico ou das enzimas que o hidroliza. A ausência de resposta positiva para a cianogênese, em certos indivíduos, pode ser devido à ausência do glicosídeo cianogênico, da hidrolase ou de ambos (Bell 2001). É interessante ressaltar que Lycopodiella cernua apresentou resultados negativos para a cianogênese durante sete meses seguidos, sendo positivo no oitavo mês analisado e negativo no mês seguinte (Tab. 2), demonstrando assim, apresentar variabilidade na produção cianogênica. É comum também, entre as espécies, a ocorrência de polimorfismo na habilidade para liberar ácido cianídrico, ou seja, dentro de determinada população pode haver indivíduos cianogênicos e outros não cianogênicos (Aikman et al. 1996).

Os meses de novembro, junho e setembro foram os que apresentaram as maiores taxas de cianogênese total no período analisado (Fig. 1). Parece não haver tendência de maior frequiência da cianogênese em determinado período do ano, como verificado por Kaplan et al. (1983) para a restinga de Maricá. Isto pode significar que o período de um ano de observações não seja suficiente para esse tipo de estudo. Contudo, os meses de dezembro e junho (sete espécies cada), setembro e novembro (seis espécies cada) apresentaram os maiores números de espécies cianogênicas (Tab. 2). Segundo Buhrmester et al. (2000), é possível ainda que alguns indivíduos que apresentaram resultado negativo tenham produzido HCN em algum outro momento do seu ciclo vital e não naquele que foram coletados para a realização dos testes. Vetter (2000) afirmou que a cianogênese é influenciada por fatores endógenos (crescimento) e exógenos (ecológicos). A natureza do substrato pode influenciar na freqüência da cianogênese nas pteridófitas, como verificado em $M$. vacciniifolia epífita e rupícola (Tab. 1 e 2) e segundo Gershenzon (1983) a deficiência de nitrogênio provoca diminuição na produção de glicosídeos cianogênicos. A pressão de insetos herbívoros pode induzir ou estimular a produção de glicosídeos cianogênicos (Kaplan et al. 1983; Larcher 2000). Cooper-Driver et al. (1977) encontraram correlação entre a produção de ácido cianídrico e a palatabilidade em Pteridium aquilinum, 


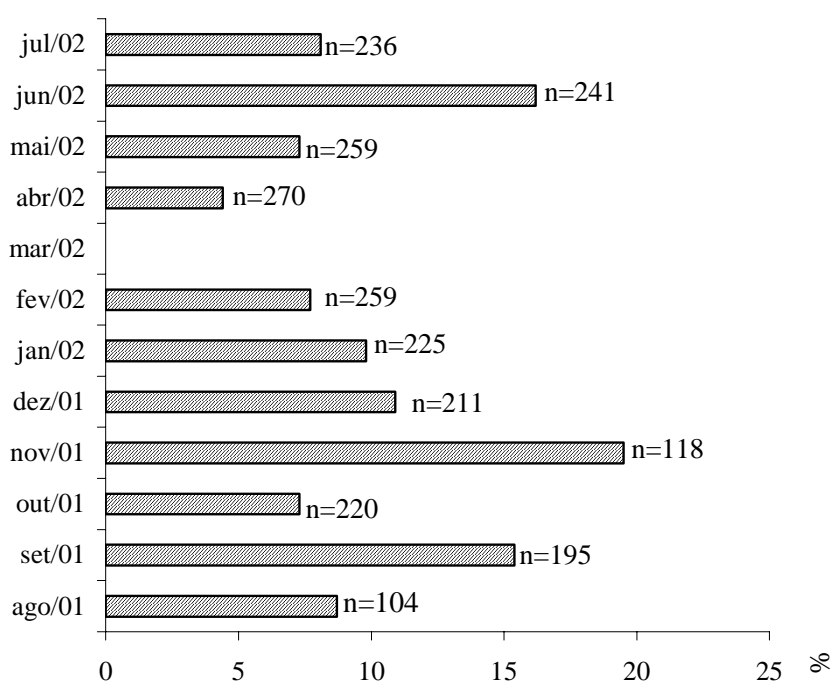

Figura 1. Percentual total mensal de amostras cianogênicas de esporófitos de pteridófitas coletados na Pedra de Itacoatiara, Niterói, RJ, Brasil, no período de agosto/2001 a julho/2002. O mês de março/2002 não foi analisado. ( $n=$ número de amostras analisadas em cada mês)

sendo a herbivoria fortemente inibida no pico da cianogênese. Alguns estudos realizados em espécies que apresentam polimorfismo na cianogênese demonstraram que os herbívoros possuíam a preferência de predar os indivíduos não cianogênicos, enquanto os cianogênicos eram evitados (CooperDriver \& Swain 1976; Bell 2001). Zagrobelny et al. (2004), no entanto, relatam que alguns herbívoros, especialmente insetos, alimentam-se preferencialmente de plantas cianogênicas. Segundo esses autores, estes herbívoros adquiriram a habilidade de metabolizar os glicosídeos cianogênicos ou seqüestrá-los para então

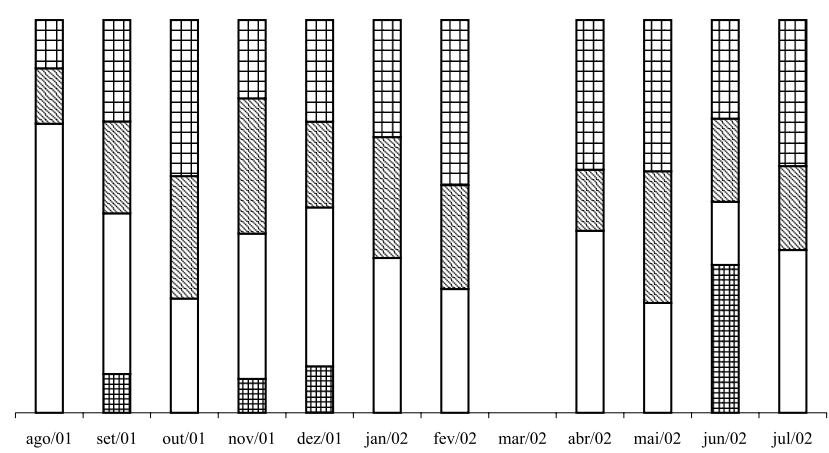

Figura 2. Produção relativa mensal (em \%) da cianogênese em caule, báculo, folha fértil e folha estéril de esporófitos de pteridófitas coletados na Pedra de Itacoatiara, Niterói, RJ, Brasil, no período de agosto/2001 e julho/2002. O mês de março/2002 não foi analisado. 嘈=Caule; $\square=$ Báculo; $=$ Folha fértil; $\boxplus=$ Folha estéril. usá-los na sua própria defesa contra predadores.

Nas espécies com resultado positivo para a cianogênese, os órgãos analisados mostraram diferentes freqüências de produção de HCN (Tab. 1; Fig. 2). O caule (exceto em junho) sempre apresentou as menores freqüências, enquanto as folhas apresentaram as maiores (Fig. 2). É importante enfatizar que nas espécies onde o caule apresentou resultado positivo para a cianogênese, este era aéreo ou a espécie epífita/rupícola (Tab. 1), o que mostra que a cianogênese, ao menos nas pteridófitas analisadas, é importante para a porção aérea do vegetal, em detrimento da subterrânea. Segundo Vetter (2000), a cianogênese pode variar em diferentes órgãos de um mesmo indivíduo e sofrer influência de fatores endógenos, como estágio de desenvolvimento. Miller et al. (2004) registraram que a intensidade luminosa interfere na alocação dos glicosídeos cianogênicos dentro do vegetal. Esses autores verificaram que sob menor intensidade luminosa, indivíduos de Prunus turneriana (F.M. Bailey) Kalkman (Rosaceae) tiveram aumento na alocação de glicosídeos cianogênicos em folhas adultas e fotossinteticamente ativas. Estudos fenológicos em pteridófitas realizados por Ranal (1995) indicaram que para algumas espécies, a periodicidade na produção de folhas parece ser função direta do fator água e que de modo geral, as folhas são produzidas após período de chuvas. Gershenzon (1983) demonstrou que o déficit hídrico aumenta a produção de glicosídeos cianogênicos. Deste modo, correlações entre estudos fenológicos e cianogênicos poderiam esclarecer a variação encontrada no presente estudo.

São estimadas para o Brasil, cerca de 1.150 espécies de pteridófitas (Windisch 1996). O presente estudo contemplou apenas 19 espécies, ou seja, apenas $1,7 \%$ do total, sendo oportuno ampliar a busca da cianogênese em outras pteridófitas. Além da necessidade do conhecimento das pteridófitas cianogênicas, inúmeros questionamentos necessitam de respostas. Quais parâmetros estão influenciando a ocorrência da cianogênese nas pteridófitas da Pedra de Itacoatiara? Quais são os glicosídeos cianogênicos, suas concentrações e importância ecológica?

\section{Referências bibliográficas}

Aikman, K.; Bergman, D.; Ebinger, J. \& Seigler, D. 1996. Variation of Cyanogenesis in Some Plant Species of the Midwestern United States. Biochemical Systematics and Ecology 24(7/8): 637-645. 
Bell, E.A. 2001. Ecological biochemistry and its development. Phytochemistry 56: 223-227.

Buhrmester, R.A.; Ebinger, J.E. \& Seigler, D.S. 2000. Sambunigrin and cyanogenic variability in populations of Sambucus canadensis L. (Caprifoliaceae). Biochemical Systematics and Ecology 28: 689-695.

Cooper-Driver, G.A. \& Swain, T. 1976. Cyanogenic polymorphism in bracken in relation to herbivore predation. Nature 260: 604.

Cooper-Driver, G.A.; Finch, S. \& Swain, T. 1977. Seasonal variation in secondary plant compounds in relation to the palatability of Pteridium aquilinum. Biochemical Systematics and Ecology 5(3): 177-183.

Gershenzon, J. 1983. Changes in the levels of plant secondary metabolites under water and nutrient stress. In: B.N. Timmermann; C. Steelink \& F.A. Loews (eds.). Phytochemical Adaptations to Stress. Recent Advances in Phytochemistry 18: $273-320$

Hadfield, P.R.H. \& Dyer, A.F. 1988. Cyanogenesis in Gametophytes and Young Sporophytes of Bracken. Biochemical Systematics and Ecology 16(1): 9-13.

Haper, N.L.; Cooper-Driver, G.A. \& Swain, T. 1976. A survey for cyanogenesis in ferns and gymnosperms. Phytochemistry 15(11): 1764-1767.

Harborne, J.B. 1984. Phytochemical methods. A guide to modern techniques of plant analysis. London, Chapman and Hall.

Jones, D.A. 1988. Cyanogenesis in animal-plant interactions. Ciba Foundation Symposium 140: 151-170.

Kaplan, M.A.C.; Figueiredo, M.R. \& Gottlieb, O.R. 1983. Variation in Cyanogenesis in Plants with Season and Insect Pressure. Biochemical Systematics and Ecology 11(4): 367-370.

Kramer, K.U. \& Green, P.S. 1990. Pteridophytes and Gymnosperms. Pp. 1-404. In: K. Kubitzki, (ed.). The families and Genera of Vascular Plants. Berlin, SpringerVerlag.

Larcher, W. 2000. Ecofisiologia vegetal. São Carlos, RiMa.

Meirelles, S.T.; Pivello, V.R. \& Joly, C.A. 1999. The vegetation of gramite rock outcrops in Rio de Janeiro, Brazil and the need por its protection. Environmental Conservation 26: 10-20.

Miller, R.E.; Gleadow, R.M. \& Woodrow, I.E. 2004. Cyanogenesis in tropical Prunus turneriana: characterisation, variation and response to low light. Functional Plant Biology 31: 491-503.
Pontes, J.A.L. 1987. Serra da Tiririca, RJ: Necessidade de Conservação (Primeira Contribuição). Boletim da Fundação Brasileira para a Conservação da Natureza 22: 89-94.

Porembski, S. \& Barthlott, W. 2000. Gramitic and gneissic outcrops (inrelbergs) as centers of diversity for dericcationtolerant vascular plants. Plant Ecology 151: 19-28.

Porembski, S. 2002. Terrestrial habitat islands as model systems for biodiversity research. Pp. 158-161. In: E.L. Araújo; A.N. Moura; E.V.S.B. Sampaio; L.M.S. Gestinari \& J.M.T. Carneiro (eds.). Biodiversidade, Conservação e Uso Sustentável da Flora do Brasil. Recife, Universidade Federal Rural de Pernambuco.

Ranal, M.A. 1995. Estabelecimento de pteridófitas em mata mesófila semidecídua do estado de São Paulo. 3. Fenologia e sobrevivência dos indivíduos. Revista Brasileira de Biologia 55(4): 777-787.

Ricklefs, R.E. 1996. A Economia da Natureza. Rio de Janeiro, Guanabara Koogan.

Schvartsman, S. 1992. Plantas venenosas e animais peçonhentos. São Paulo, Sarvier.

Silva, A.T. 1984. Pteridófitas. Pp. 33-34. In: O. Fidalgo \& V.L.R. Bononi (coords.). Técnicas de coleta, herborização e preservação de material botânico. São Paulo, Instituto de Botânica.

Swain, T. \& Cooper-Driver, G. 1973. Biochemical systematics in the Filicopsida. In: A.C. Jermy, J.A. Crabbe \& B.A. Thomas (eds.). The phylogeny and classification of the ferns. Botanical Journal of the Linnean Society (supplement 1) 67: 111-134.

Tryon, R.M. 1986. The Biogeography of Species, with Special Reference to Ferns. The Botanical Review 52(2): 117-155.

Tryon, R.M. \& Tryon, A.F. 1982. Ferns and allied Plants with Special reference to Tropical America. New York, Springer-Verlag.

Windisch, P.G. 1992. Pteridófitas da região Norte-ocidental do Estado de São Paulo (Guia para estudo e excursões). São José do Rio Preto, Universidade Estadual Paulista.

Windisch, P.G. 1996. Towards assaying biodiversity in brazilian pteridophytes. Pp. 109-117. In: C.E. Bicudo \& N.A. Menezes (eds.). Biodiversity in Brazil a first approach. Brasília, Conselho Nacional de Desenvolvimento Científico e Tecnológico.

Vetter, J. 2000. Plant cyanogenic glycosides. Toxicon 38: 11-36. Zagrobelny, M.; Bak, S.; Rasmussen, A.V.; Jørnensen, B.; Naumann, C.M. \& Møller, B.L. 2004. Cyanogenic glucosides and plant-insect interactions. Phytochemistry 65: 293-306. 